\title{
Proficiency Testing of
} Metagenomics-Based Detection of Food-Borne Pathogens Using a Complex Artificial Sequencing Dataset

\section{OPEN ACCESS}

David Rodriguez-Lazaro, University of Burgos, Spain

Reviewed by:

Laurent Dacheux, Institut Pasteur, France

Samantha Chandranath Karunarathna,

Kunming Institute of Botany, China

*Correspondence: Dirk Höper Dirk.Hoeper@fli.de

Specialty section: This article was submitted to Food Microbiology, a section of the journal

Frontiers in Microbiology

Received: 23 June 2020 Accepted: 13 October 2020 Published: 04 November 2020

Citation:

Höper D, Grützke J, Brinkmann A, Mossong J, Matamoros S, Ellis RJ,

Deneke C, Tausch SH, Cuesta I,

Monzón S, Juliá M, Petersen TN,

Hendriksen RS, Pamp SJ, Leijon M, Hakhverdyan M, Walsh AM,

Cotter PD, Chandrasekaran L,

Tay MYF, Schlundt J, Sala C,

De Cesare A, Nitsche A, Beer M and Wylezich C (2020) Proficiency Testing of Metagenomics-Based Detection of Food-Borne Pathogens Using

a Complex Artificial Sequencing Dataset. Front. Microbiol. 11:575377.

doi: 10.3389/fmicb.2020.575377
Dirk Höper ${ }^{*}$, Josephine Grützke ${ }^{2}$, Annika Brinkmann³ ${ }^{3}$ Joël Mossong ${ }^{4}$, Sébastien Matamoros ${ }^{5}$, Richard J. Ellis ${ }^{6}$, Carlus Deneke ${ }^{2}$, Simon H. Tausch ${ }^{2}$, Isabel Cuesta7, Sara Monzón7, Miguel Juliá7, Thomas Nordahl Petersen ${ }^{8}$, Rene S. Hendriksen ${ }^{8}$, Sünje J. Pamp ${ }^{8}$, Mikael Leijon ${ }^{9}$, Mikhayil Hakhverdyan ${ }^{9}$, Aaron M. Walsh ${ }^{10}$, Paul D. Cotter ${ }^{10}$, Lakshmi Chandrasekaran ${ }^{11}$, Moon Y. F. Tay ${ }^{11}$, Joergen Schlundt ${ }^{11}$, Claudia Sala ${ }^{12}$, Alessandra De Cesare ${ }^{13}$, Andreas Nitsche ${ }^{3}$, Martin Beer ${ }^{1}$ and Claudia Wylezich ${ }^{1}$

\footnotetext{
${ }^{1}$ Institute of Diagnostic Virology, Friedrich-Loeffler-Institut, Greifswald-Insel Riems, Germany, ${ }^{2}$ Department of Biological Safety, German Federal Institute for Risk Assessment, Berlin, Germany, ${ }^{3}$ Centre for Biological Threats and Special Pathogens, Robert Koch Institute, Berlin, Germany, ' Département de Microbiologie, Laboratoire National de Santé, Dudelange, Luxembourg, ${ }^{5}$ Department of Medical Microbiology, Amsterdam UMC University of Amsterdam, Amsterdam, Netherlands, ${ }^{6}$ Animal and Plant Health Agency, Addlestone, United Kingdom, ${ }^{7}$ Bioinformatics Unit, Institute of Health Carlos III (ISCIII), Madrid, Spain, ${ }^{8}$ Research Group for Genomic Epidemiology, National Food Institute, Technical University of Denmark, Lyngby, Denmark, ${ }^{9}$ Department of Microbiology, National Veterinary Institute (SVA), Uppsala, Sweden,

${ }^{10}$ Teagasc Food Research Centre, APC Microbiome Ireland and Vistamilk, Moorepark, Ireland, ${ }^{11}$ Nanyang Technological University Food Technology Centre (NAFTEC), Nanyang Technological University (NTU), Singapore, Singapore,

${ }^{12}$ Department of Physics and Astronomy, University of Bologna, Bologna, Italy, ${ }^{13}$ Department of Veterinary Medical Sciences, University of Bologna, Bologna, Italy
}

Metagenomics-based high-throughput sequencing (HTS) enables comprehensive detection of all species comprised in a sample with a single assay and is becoming a standard method for outbreak investigation. However, unlike real-time PCR or serological assays, HTS datasets generated for pathogen detection do not easily provide yes/no answers. Rather, results of the taxonomic read assignment need to be assessed by trained personnel to gain information thereof. Proficiency tests are important instruments of validation, harmonization, and standardization. Within the European Union funded project COMPARE [COllaborative Management Platform for detection and Analyses of (Re-) emerging and foodborne outbreaks in Europe], we conducted a proficiency test to scrutinize the ability to assess diagnostic metagenomics data. An artificial dataset resembling shotgun sequencing of RNA from a sample of contaminated trout was provided to 12 participants with the request to provide a table with per-read taxonomic assignments at species level and a report with a summary and assessment of their findings, considering different categories like pathogen, background, or contaminations. Analysis of the read assignment tables showed that the software used reliably classified the reads taxonomically overall. However, usage of incomplete reference databases or inappropriate data pre-processing caused difficulties. From the combination of the participants' reports with their read assignments, we conclude that, although most species were detected, a number of 
important taxa were not or not correctly categorized. This implies that knowledge of and awareness for potentially dangerous species and contaminations need to be improved, hence, capacity building for the interpretation of diagnostic metagenomics datasets is necessary.

Keywords: background contamination, diagnostic assessment, high-throughput sequencing, metagenomics, pathogen, proficiency test, training

\section{INTRODUCTION}

Approaches for the investigation of food-borne outbreaks regarding pathogen characterization, source attribution and risk assessment need to be precise, fast and independent from slow and biased cultivation techniques. Metagenomics-based highthroughput sequencing (HTS) is becoming a standard method for outbreak investigations of non-culturable, difficult-to-culture or slow-growing microorganisms (Koutsoumanis et al., 2019) yet protocols and analysis pipelines need to be standardized for routine use. In addition, training in result assessment and interpretation is needed for unexperienced users to be applicable as gold standard.

Problems with the analysis and the diagnostic assessment of HTS datasets may occur in several sample processing steps including sequencing, and during bioinformatics analysis. Beside the contamination of a sample during sampling and sample processing, microbial DNA can be introduced within the reagents during the preparation of sequencing libraries (Salter et al., 2014). Therefore, the specific reagent background should be known in ideal circumstances (Kirstahler et al., 2018; Wylezich et al., 2018) and at the very least, should be taken into consideration when assessing the taxa found in metagenomics datasets. Cross-contamination of multiplexed libraries is possible due to adapter swapping (Sinha et al., 2017) or carry-over between runs (Illumina, 2013; Höper et al., 2016). Furthermore, the interpreter of data should be aware of possible false-positives detected due to contaminated genomes and insufficiently curated databases (e.g., Kirstahler et al., 2018). All these points are very important when interpreting metagenomic datasets in search for possible pathogens that may be less abundant in terms of sequencing reads.

Some ring trials and proficiency tests have previously been conducted to push forward the standardization of HTS approaches and their implementation into clinical diagnostic routine and diagnosis reporting systems. Metagenomics-based proficiency tests for pathogen detection have often been focused on virus detection (Brinkmann et al., 2019; Junier et al., 2019; Zamperin et al., 2019). These studies highlighted that recognition of viruses that exhibit high mutation rates can cause some difficulties and might only be possible by inclusion of protein-based alignment approaches (Brinkmann et al., 2019). In addition, the undetected pathogen of relevance can also be a bacterial or parasite pathogen, which could be comprised in the sample but be masked by the sample background. Thus, interpreters need to train their capability recognizing the different categories of contaminations.
To some extent, the wet lab procedures for metagenomicsbased pathogen detection were already standardized within the European Union funded COMPARE-network. ${ }^{1}$ Within this framework, a first metagenomics-based virus detection proficiency test with separate parts addressing bioinformatics only or sample processing combined with bioinformatics and result assessment, respectively, was conducted (Brinkmann et al., 2019). Due to the importance of foods in the transmission of zoonotic agents, a second proficiency test for metagenomicsbased pathogen detection in food was organized in 2018 within the COMPARE network. Again, this proficiency test consisted of two independent parts addressing either the sample processing (wet lab) or bioinformatics combined with result assessment (dry lab). In the laboratory part (wet lab), the participants had to generate a metagenomic sequence dataset from a piece of smoked salmon that was spiked with a complex mock community (bacteria, fungi, eukaryotic parasite, and virus). The wet lab part was analyzed and is published separately in a companion manuscript (Sala et al., Unpublished). In the present study, the bioinformatics and assessment part (dry lab), the participants had to analyze a synthetic dataset and assess their obtained result. While here the analysis of the same dataset by all participants ensured the comparability of the submitted results, in the wet lab part, the comparison of the wet lab workflows was ensured by the centralized sequence data analysis. Moreover, participants were free to decide in which of the two independent parts they participated.

Whereas in the first COMPARE virus proficiency test (Brinkmann et al., 2019) the quality of the data analysis software was in the focus, in the present proficiency test we focused on the participants assessment of the results obtained by the software. Hence, the purpose was (i) testing the interpretation of results obtained from the software analysis by the participants, i.e., the recognition of potentially dangerous species and (ii) the awareness of artifacts occurring in the sample processing and sequencing.

\section{MATERIALS AND METHODS}

\section{Organization of the Dry Lab Proficiency Test}

Like the COMPARE virus proficiency test (Brinkmann et al., 2019), the food metagenomics proficiency test dry lab part was initiated within the COMPARE network, and

\footnotetext{
${ }^{1}$ https://www.compare-europe.eu/library/protocols-and-sops
} 
arranged by the network partner Friedrich-Loeffler-Institut between April and June 2018. The participants of the dry lab part of the food metagenomics proficiency test received a synthetically generated complex metagenomics sequencing dataset that they had to analyze and assess their obtained results regarding potentially present pathogens, sequencing artifacts, potential lab contaminations, and other information deemed relevant for assessment.

\section{Participants of the Dry Lab Part}

Twelve participants applied for the COMPARE food metagenomics dry lab proficiency test and completed the survey. Participants were registered from Denmark $(n=1)$, Germany $(n=3)$, Luxembourg $(n=1)$, Ireland $(n=1)$, Italy $(n=1)$, Netherlands $(n=1)$, Singapore $(n=1)$, Spain $(n=1)$, Sweden $(n=1)$, and United Kingdom $(n=1)$. The 12 participants represented 12 different institutes or organizations. Information about the participants' background is given in Table 2 .

\section{Creating a Synthetic Metagenomics Dataset}

The synthetic metagenomics dataset was created using ART_Illumina, Q Version 2.5.8 (Huang et al., 2012) and a number of EST (expressed sequence tag) and coding sequence data retrieved from the NCBI database (see Table 1). For details of the dataset creation, please refer to Supplementary Materials and Methods.

\section{Requirements to Participants}

In parallel with the dry lab synthetic dataset, an email (see email 1 in the Supplementary Materials and Methods) was sent to all participants that included the necessary instructions for the data and assessment preparation and delivery. The requirements were later further specified (see email 2 in the Supplementary Materials and Methods). Briefly, the participants were asked to use their routine bioinformatics for taxonomic read classification and to submit a table in which the taxonomic classification for all reads was recorded in two columns ("Read accession" and "Species"). Hereinafter, this table is called "read assignment table." Furthermore, they were asked to prepare a report as Word file or pdf containing their summary and assessment of the read classifications. They were asked to especially consider potentially present pathogens, sequencing artifacts, possible sequencing lab contaminations, and other facts they deemed important. This document is called "summary and assessment file" in the following.

The participants had about 50 days (25 April-15 June 2018) for conducting a bioinformatics analysis with the dataset provided and for assessing the results from obtained data until the delivery of their results.

\section{Data Analyses}

The submitted read assignment tables were analyzed using $\mathrm{R}$ and RStudio ( $\mathrm{R}$ v3.6.2, $\mathrm{R}$ Core Team, 2019; RStudio v1.2.5033). ${ }^{2}$ For an assessment of the taxonomic classifications reported in the participants' read assignment tables, sensitivity,

${ }^{2}$ https://rstudio.com/ specificity, correct classification rate, positive predictive value, and negative predictive value were calculated from the readto-taxon assignments. For further details of these calculations, please see Supplementary Materials and Methods. For the final rating of the participants' assessments, the expected assessments were defined as shown in Table 1. The submitted assessments were rated in five different classes: (i) species detected and rated by the participant as expected, (ii) species detected and rated more serious than expected, (iii) species detected but assessed less critical than expected, (iv) species detected but not assessed, and (v) species not detected (according to the submitted read assignment table).

\section{Data Availability}

The synthetic metagenomics dataset simulating a contaminated trout was submitted to European Nucleotide Archive and is accessible under the study accession number PRJEB37463. The read-accessions in this file comprise the database identifiers of the used reference sequence and the suffix "-fpt2018" with a continuous numbering per input sequence, for instance “lc1|NC_026023.1_cds_YP_009113336.1_1-fpt2018105”.

\section{RESULTS AND DISCUSSION}

The aim of the present proficiency test was in particular to test the interpretation of results obtained from metagenomics sequencing datasets based on the software analysis performed by the participants. For this purpose, the detection of potentially dangerous species was most important but also the awareness and recognition of artifacts that may occur during the wet lab sample processing and sequencing.

\section{General Considerations of the Provided Files}

All participants except one (P10) uploaded the requested read assignment table with the read to species assignments output by their applied software pipeline, eight of which in due time, and three with a delay. All participants sent in a summary and assessment file of their obtained results (see Supplementary Material).

The format requested for the preparation of the read assignment tables (see above and in Supplementary Materials and Methods) turned out to be not clear for all participants. Six participants sent in incomplete tables, only reporting assignments for a small fraction of reads (see Figure 1). Moreover, only five participants sent in their read assignment tables in the requested format. In the remaining cases, participants reported more details with strain or isolate names or incomplete assignments only to a level between superkingdom and genus. Especially the users of Kraken did not report in the requested format but used the Kraken format including the full taxonomic path up to the most specific level reported. A similar observation was recently made by the organizers of another ring trial, who stated that the participants did not in all cases use official scientific names. They emphasized the importance of using a standardized set of species names based on NCBI taxonomy for reporting (Junier et al., 2019). 
TABLE 1 | Composition of the simulated sequence dataset.

\begin{tabular}{|c|c|c|c|c|}
\hline Species & Super-Kingdom & Category & Rationale & Number Reads \\
\hline Bacteroides fragilis & Bacteria & Opportunistic pathogen & Analogous to wet-lab proficiency test mock community & 20,000 \\
\hline Burkholderia pseudomallei & Bacteria & Pathogen & $\begin{array}{l}\text { Burkholderiaceae also found in real smoked salmon } \\
\text { sample }\end{array}$ & 8,000 \\
\hline Escherichia coli & Bacteria & Pathogen & $\begin{array}{l}\text { Analogous to wet-lab proficiency test mock community, } \\
\text { also found in real smoked salmon sample }\end{array}$ & 80,000 \\
\hline Salmonella enterica & Bacteria & Pathogen & Analogous to wet-lab proficiency test mock community & 125,000 \\
\hline Fusobacterium nucleatum & Bacteria & Opportunistic pathogen & Analogous to wet-lab proficiency test mock community & 40,000 \\
\hline Lactobacillus acidophilus & Bacteria & Background & Food additive & 175,000 \\
\hline Lactobacillus delbrueckii & Bacteria & Background & & 25,000 \\
\hline Listeria monocytogenes & Bacteria & Pathogen & Frequently found as food contamination & 10,000 \\
\hline Mycobacterium colombiense & Bacteria & Opportunistic pathogen & Mycobacteria with increasing impact as food spoilage & 2,000 \\
\hline Pseudomonas libanensis & Bacteria & Background & $\begin{array}{l}\text { P. libanensis previously detected in food; } \\
\text { Pseudomonadaceae also found in real smoked salmon } \\
\text { sample }\end{array}$ & 200 \\
\hline Anisakis berlandi & Eukaryota & Pathogen & $\begin{array}{l}\text { Analogous to Cryptosporidium parvum from wet-lab } \\
\text { proficiency test mock community }\end{array}$ & 1,212 \\
\hline Anisakis brevispiculata & Eukaryota & Pathogen & & 246 \\
\hline Anisakis paggiae & Eukaryota & Pathogen & & 248 \\
\hline Anisakis pegreffii & Eukaryota & Pathogen & & 2,051 \\
\hline Anisakis physeteris & Eukaryota & Pathogen & & 262 \\
\hline Anisakis simplex & Eukaryota & Pathogen & & 6,044 \\
\hline Anisakis typica & Eukaryota & Pathogen & & 247 \\
\hline Aspergillus flavus & Eukaryota & Opportunistic pathogen & $\begin{array}{l}\text { Analogous to Saccharomyces cerevisiae from wet-lab } \\
\text { proficiency test mock community, toxin producer }\end{array}$ & 5,000 \\
\hline Danio rerio & Eukaryota & Database misclassification & & 4,310 \\
\hline Brugia malayi & Eukaryota & & & 64 \\
\hline Caenorhabditis remanei & Eukaryota & & & 193 \\
\hline Scomber japonicus & Eukaryota & & & 181 \\
\hline Oncorhynchus mykiss & Eukaryota & Food & O. mykiss EST data as host background & $9,451,675$ \\
\hline African swine fever virus (ASFV) & Viruses & Run contamination & $\begin{array}{l}\text { DNA virus; barcode mis-alignment/index swapping on } \\
\text { Illumina MiSeq }\end{array}$ & 15 \\
\hline Norwalk virus & Viruses & Pathogen & RNA virus; typical food contaminant & 946 \\
\hline Escherichia virus phiX174 (phiX174) & Viruses & Run contamination & $\begin{array}{l}\text { Barcode mis-alignment/index swapping on Illumina } \\
\text { MiSeq }\end{array}$ & 735 \\
\hline $\begin{array}{l}\text { Aspergillus foetidus dsRNA } \\
\text { mycovirus (AfdsV) }\end{array}$ & Viruses & Background & Model for virus of the contaminant Aspergillus flavus & 107 \\
\hline Sum & & & & $9,958,736$ \\
\hline
\end{tabular}

The summary and assessment files of four participants (P2, P4, P5, and P10) only contained the requested summary table without an assessment. The remaining eight participants provided both the requested summary table and an assessment of the reported results.

\section{Insights From Analysis of the Read Assignment Tables}

Of the uploaded 11 read assignment tables, only five (from P1, P3, P4, P8, and P11) contained an assignment for all reads of the dataset; the remaining six contained assignments for approximately $3-18 \%$ of the reads (Figure 1 ). The reasons not to report assignments for the missing reads were (i) filtered low quality (P2, P6, P7, and P9), (ii) filtered eukaryotic sequences (P2, P5, P6, and P9), or (iii) no justification (P12). In two cases (P1 and P3; see Figure 1) the majority of the reads (approximately 95\%) was reported "unclassified." In one case (P1), this was partly due to incomplete classification to higher-level taxonomic entities only. Three participants ( $\mathrm{P} 4, \mathrm{P} 8$, and $\mathrm{P} 11$; Figure 1) reported classifications for the majority of the reads and only in these cases the overall composition resembled the actual one (Figure 1).

The provided read assignments were used to calculate the key characteristics of the classifications. Namely, specificity, correct classification rate, and negative predictive values (Supplementary Figures 1-3), as well as positive predictive value (Figure 2) and sensitivity (Figure 3) were determined. While the specificity, correct classification rate (except for host sequences), 
TABLE 2 | Participants, their background and applied data processing and important comments from their summaries.

\begin{tabular}{|c|c|c|c|}
\hline Participants & $\begin{array}{l}\text { Participants' } \\
\text { sector }\end{array}$ & Data processing workflow & Participants comments \\
\hline $\mathrm{P} 1$ & Food & $\begin{array}{l}\text { - Quality assessment w/FastQC (Wingett and Andrews, 2018) } \\
\text { - Quality trimming w/fastp (Chen et al., 2018) } \\
\text { - Taxonomic classification w/Kraken (custom database and } \\
\text { MiniKraken DB; Wood and Salzberg, 2014) } \\
\text { - Additionally pathoLive analysis for classification of viral reads } \\
\text { (Tausch et al., 2018) }\end{array}$ & $\begin{array}{l}\text { - FastQC revealed bases of bad quality at the beginning of the } \\
\text { reads. Therefore the reads were trimmed } \\
\text { - kraken analysis with custom database resulted in many } \\
\text { false-positive results; therefore, results were confirmed with } \\
\text { BLASTn (Boratyn et al., 2012). }\end{array}$ \\
\hline P4 & Food & $\begin{array}{l}\text { - Quality assessment w/FastQC (Wingett and Andrews, 2018) } \\
\text { - Quality/Adapter trimming w/BBduk v. } 36.49 \\
\text { (https://sourceforge.net/projects/bbmap/) } \\
\text { - Taxonomic classification w/MGmapper (Petersen et al., 2017) }\end{array}$ & $\begin{array}{l}\text { - mapped against a phiX174 reference sequence to remove } \\
\text { potential control library reads } \\
\text { - Certain contaminations can be difficult to identify without control } \\
\text { samples. For example, certain microorganisms may be part of } \\
\text { the natural microbiome of fish or could have been introduced } \\
\text { during sample handling and processing. }\end{array}$ \\
\hline P5 & Veterinary & $\begin{array}{l}\text { - Quality assessment w/FastQC (Wingett and Andrews, 2018) } \\
\text { - Quality/Adapter trimming w/Trimmomatic (Bolger et al., 2014) } \\
\text { - Host sequence removal w/BWA-MEM (Li, 2013) } \\
\text { - Taxonomic classification w/Kraken (MiniKraken database; ref. } \\
\text { Wood and Salzberg, 2014) }\end{array}$ & $\begin{array}{l}\text { - Filtered for minimum read count (threshold } 500 \text { reads) } \\
\text { - species for which there were less than 1,000 reads would need } \\
\text { further confirmation before release of the information } \\
\text { - PhiX carry over from the sequencing lab }\end{array}$ \\
\hline P7 & Human & $\begin{array}{l}\text { - Quality/Adapter trimming w/Trimmomatic (Bolger et al., 2014) } \\
\text { - Host sequence removal w/Bowtie2 (Langmead and Salzberg, } \\
\text { 2012) } \\
\text { - Taxonomic classification w/MALT (Herbig et al., 2016), } \\
\text { DIAMOND (Buchfink et al., 2015), MEGAN (Huson et al., 2016), } \\
\text { custom database with refseq viruses, bacterial, fungi, and } \\
\text { protists }\end{array}$ & $\begin{array}{l}\text { - Pathogen of importance is Norovirus GV } \\
\text { - Abundance of a cloning vector could be an artifact of } \\
\text { sequencing reagents and preparation }\end{array}$ \\
\hline P8 & Veterinary & $\begin{array}{l}\text { - Quality trimming w/RIEMS (Scheuch et al., 2015) } \\
\text { - Taxonomic classification w/RIEMS (Scheuch et al., 2015); ncbi } \\
\text { nt }\end{array}$ & $\begin{array}{l}\text { - The Calicivirdae/Norwalk virus reads indicate the presence of } \\
\text { noroviruses in the sample. This is the most important } \\
\text { entero-pathogenic virus in the analyzed sample }\end{array}$ \\
\hline P9 & Food & $\begin{array}{l}\text { - Species-level classification w/Kraken (Wood and Salzberg, } \\
\text { 2014) } \\
\text { - Multi-locus sequence types (MLSTs) reconstructed } \\
\text { W/MetaMLST (Zolfo et al., 2017) } \\
\text { - Strain-level identification w/PanPhIAn (Scholz et al., 2016) }\end{array}$ & None \\
\hline P10 & Food & $\begin{array}{l}\text { - Quality assessment w/FastQC (Wingett and Andrews, 2018) } \\
\text { - Quality trimming w/cutadapt (part of MGmapper processing; } \\
\text { (Martin, 2011) } \\
\text { - Taxonomic classification w/MGmapper (Petersen et al., 2017) }\end{array}$ & $\begin{array}{l}\text { - no hits w/default settings, re-analysis w/adjusted parameters } \\
\quad \text { (max mismatch ratio }=0.15 \text {, min read count }=20) \\
\text { - sequence GC content measured by FastQC is reported as } \\
\text { failure }\end{array}$ \\
\hline
\end{tabular}


TABLE 2 | Continued

\begin{tabular}{|c|c|c|c|}
\hline Participants & Participants' sector & Data processing workflow & Participants comments \\
\hline P11 & Veterinary & $\begin{array}{l}\text { - Host sequences were removed by blasting (BLASTn) against a database } \\
\text { created from the Oncorhynchus mykiss isolate Swanson WGS data (NCBI acc. } \\
\text { MSJN00000000.1) using an E-value cutoff 1E-100 } \\
\text { - Taxonomic classification w/carried out by blasting (BLASTn) the remaining reads } \\
\text { against an NCBI nt database using TimeLogic }{ }^{\circledR} \text { DeCypher }{ }^{\circledR} \text { server (Active Motif } \\
\text { Inc., Carlsbad, CA, United States) with an E-value cutoff of 1E-5. The } \\
\text { assignment of sequences to species were carried out by an in-house Python } \\
\text { script using the nucl_gb.accessions2taxid (accession to taxid) and names.dmp } \\
\text { (taxid to scientific names) files available from the resources at NCBI }\end{array}$ & $\begin{array}{l}\text { - We included Murine norovirus in the } \\
\text { table despite it is not human pathogen }\end{array}$ \\
\hline P12 & Veterinary/Food & $\begin{array}{l}\text { - Quality assessment w/FastQC (Wingett and Andrews, 2018) } \\
\text { - Quality/Adapter trimming w/cutadapt (part of MGmapper processing) (Martin, } \\
\text { 2011) Taxonomic classification w/MGmapper (Databases: Bacteria, } \\
\text { Bacteria_draft, Human Microbiome, Virus, Fungi, Protozoa,and } \\
\text { MetaHitAssembly) (Petersen et al., 2017) }\end{array}$ & None \\
\hline
\end{tabular}

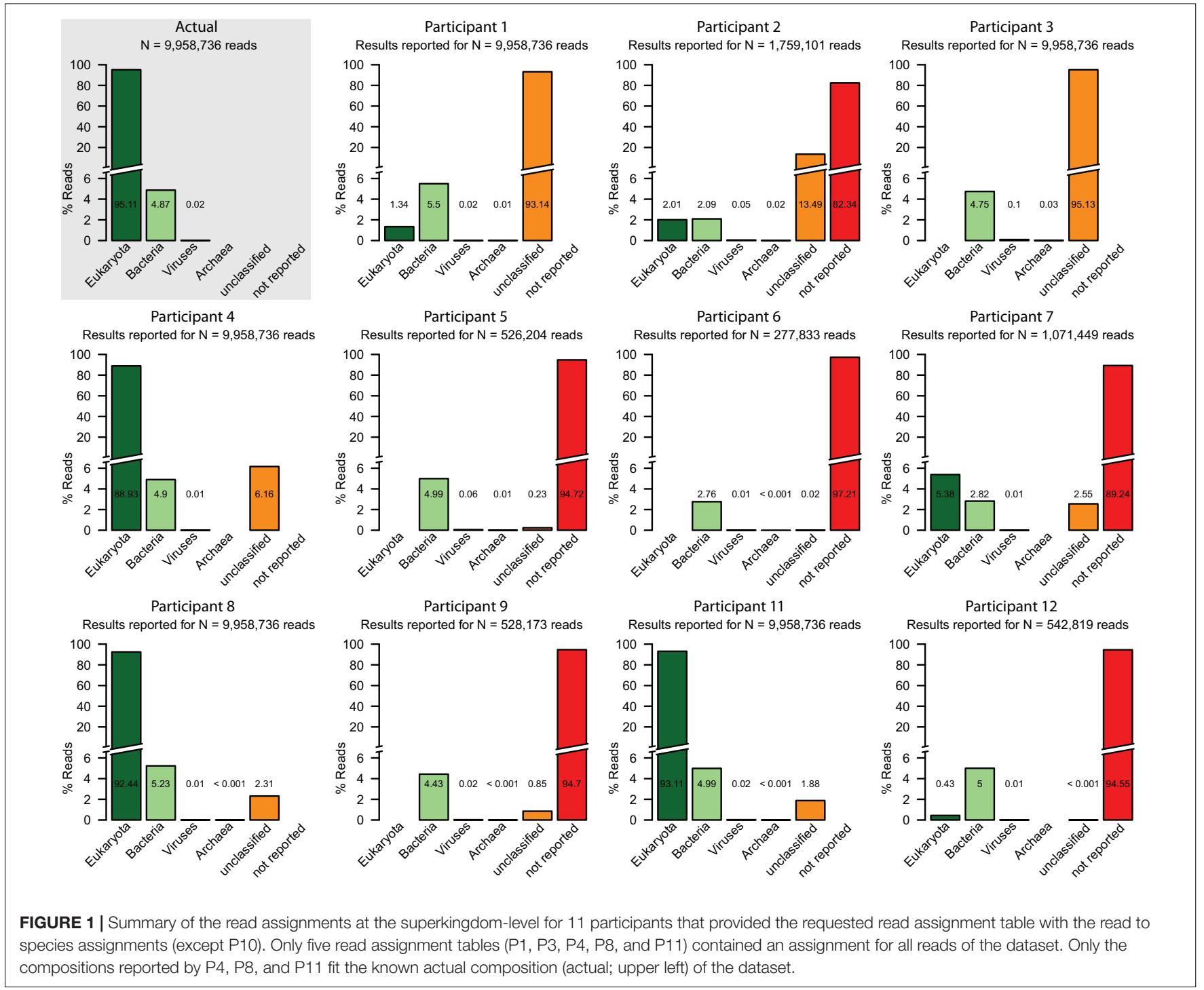

and negative predictive value (except for host sequences) were in all analyses high, the positive predictive value (Figure 2) and especially the sensitivity (Figure 3) were in some cases insufficient. Both the positive predictive value and the sensitivity appear to be compromised by either the use of incomplete databases for the taxonomic classification and/or by improper 

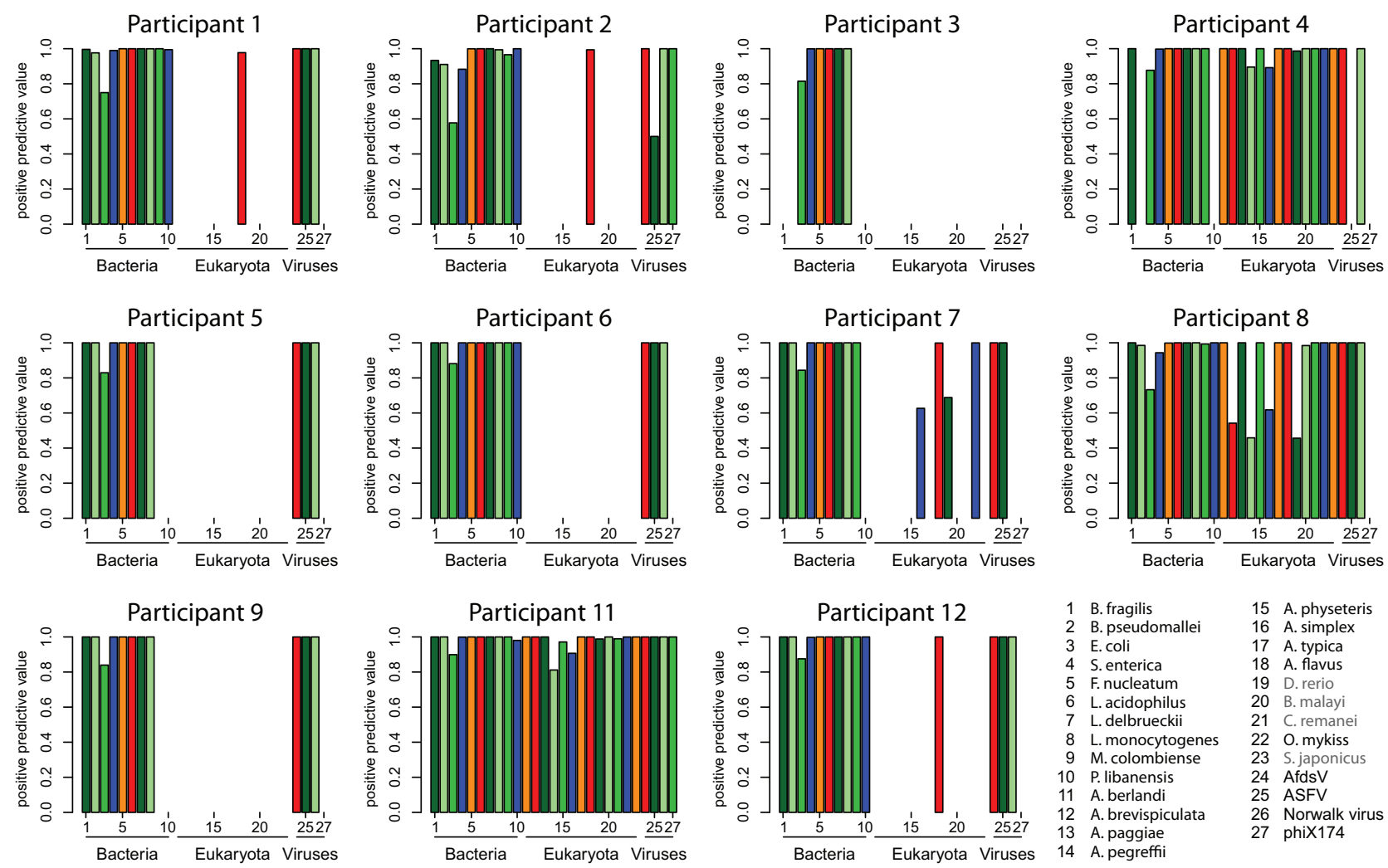

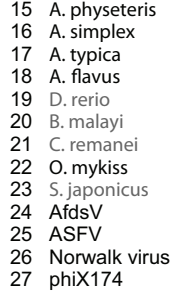

FIGURE 2 | Positive predictive values of read assignments calculated from the complete read set, calculated based on the species assignments. Sequences of the taxa labeled gray (Brugia malayi, Caenorhabditis remanei, Danio rerio, and Scomber japonicus) were downloaded unintentionally as part of the Anisakis sequence dataset.

pre-processing of the dataset (compare data analysis workflows and comments in Table 2). In three cases (P2, P6, and P7), pre-processing using software default or otherwise accepted parameters appears to have removed reads by chance, because the sensitivity is at the same low level for all detected species, meaning that this was not a species-specific effect (see results for participants P2, P6, and P7 in Figure 3). The high rate of discarded reads could have prevailed the respective participants to have a closer look at the reason for this phenomenon and modify the software settings depending on the dataset quality. The notion that this was not a species-specific effect is also emphasized by the high specificity mentioned above and by the unaltered sensitivity in case of calculation at the genus, instead the species level (see Figure 3B). If it was a species-specific effect, this should result in an improved sensitivity because then reads that are classified to closely related species should improve the result, as can indeed be seen by comparison of results obtained for participants P8 and P11 (compare Figure 3B). This was possibly the case with Pseudomonas libanensis in the results of participants P3, P4, P7, and P9 (Figure 3B).

Despite the partly suboptimal results calculated from the submitted complete classifications, Figure $4 \mathrm{~A}$ clearly demonstrates that in most cases the majority of the comprised species were detected by the used software. A marked exception were those species that were not deliberately included in the dataset, namely Scomber japonicus, Brugia malayi, Danio rerio, and Caenorhabditis remanei. These were only detected by three participants (P4, P8, and P11) who used (nearly) complete databases for the taxonomic classification. All other participants reported to have used custom databases or the MiniKraken database that also comprises only selected sequences (Wood and Salzberg, 2014). This database effect is also highlighted by the fact that at least seven and five participants, respectively, failed to detect Anisakis simplex and Aspergillus flavus, two eukaryotic taxa. This emphasizes the impact of the database used for taxonomic classification to obtain a comprehensive classification. This result is in contrast to the results of another proficiency test (Junier et al., 2019), where the impact of the database was negligible compared with the influence of the applied algorithms.

\section{Insights From Evaluations of Participants' Summary and Assessment Files}

In the present proficiency test, the assessment of the results turned out to be the most critical part. Overall, the quality of the reports was varying. Although explicitly requested, the results of the taxonomic binning were not in all cases assessed regarding the requested categories (potentially present pathogens, sequencing artifacts, possible sequencing lab contaminations, and other important facts). Looking at the summary and assessment files, there was no correlation between the overall quality of the 

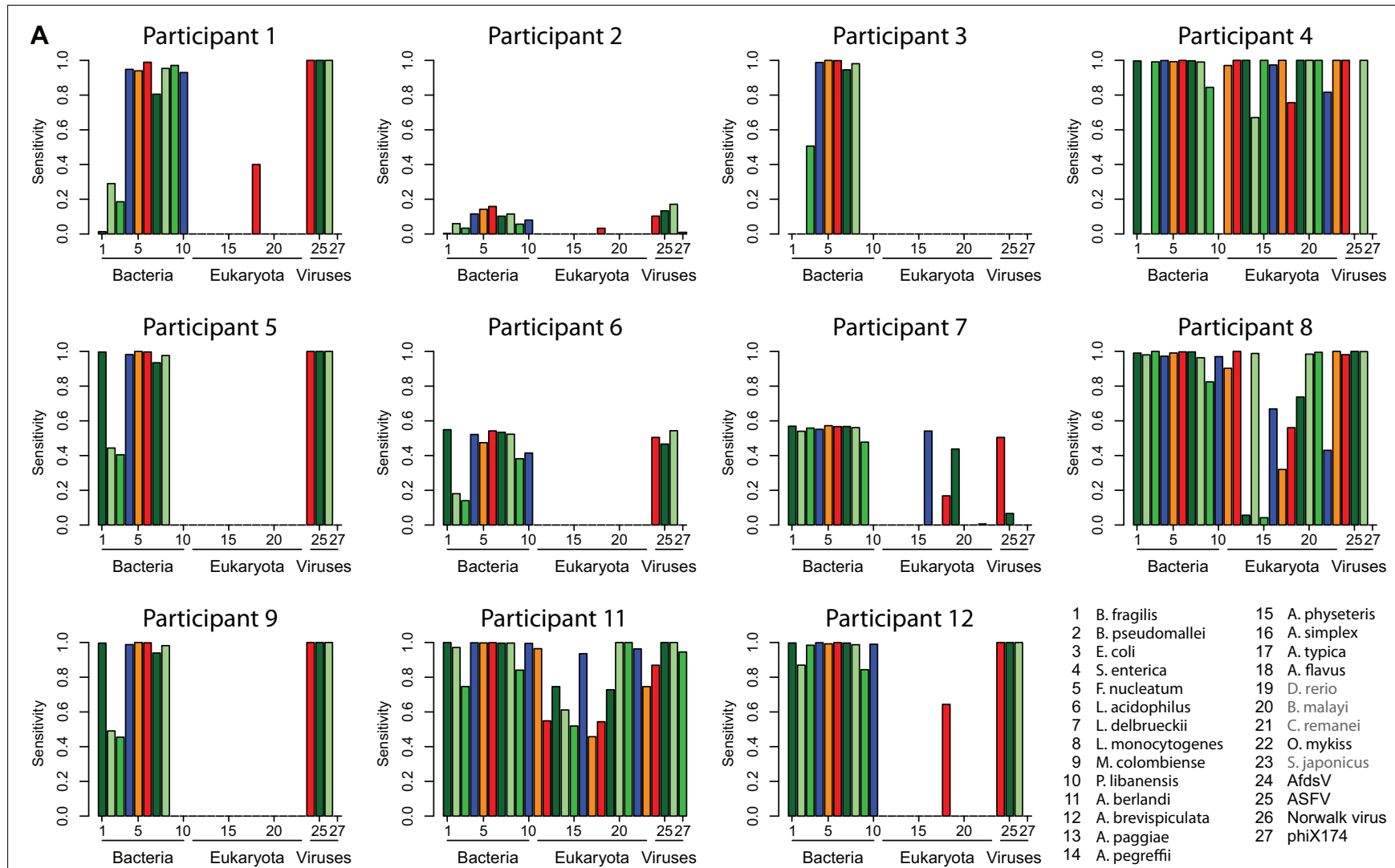
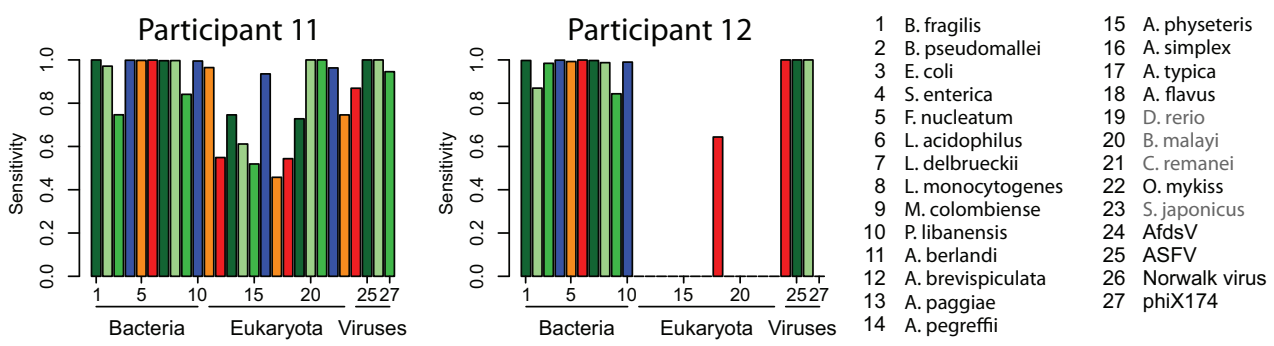

Participant 3

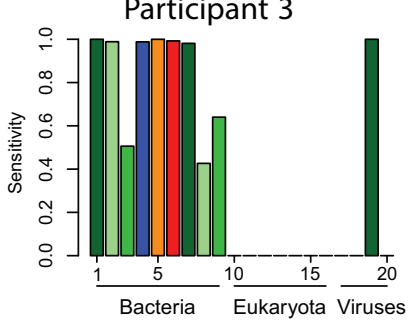

Participant 8

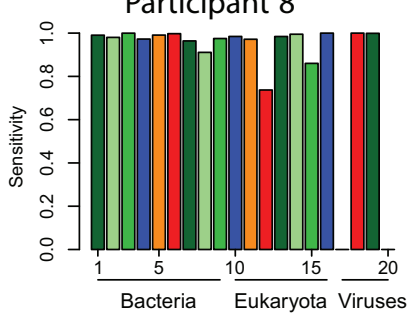

9 Pseudomonas

10 Anisakis

11 Aspergillus

12 Danio
Participant 4

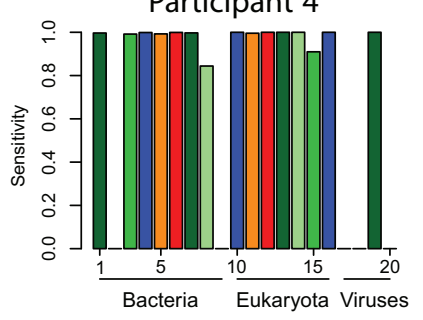

Participant 9

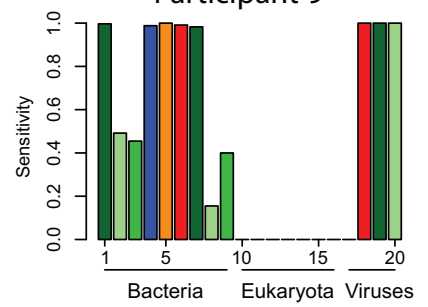

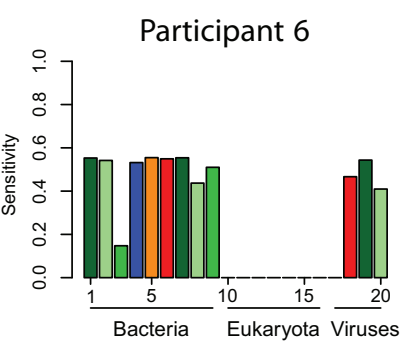

Participant 11

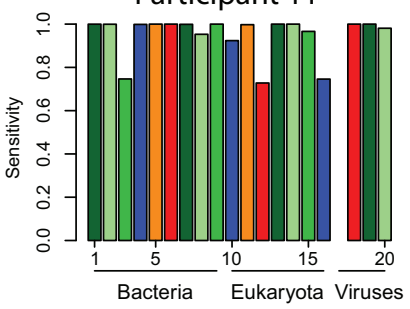

13 Brugia

14 Caenorhabditis 15 Oncorhynchus 16 Scomber

$$
17 \text { unclassified }
$$$$
19 \text { Norovirus }
$$

20 Phix174microvirus

FIGURE 3 | Sensitivity of read assignments calculated from the complete read set. (A) Sensitivities calculated based on the species assignments. (B) Sensitivities calculated based on the genus assignments. Sequences of the taxa labeled gray (Brugia malayi, Caenorhabditis remanei, Danio rerio, and Scomber japonicus) were downloaded unintentionally as part of the Anisakis sequence dataset. 
A

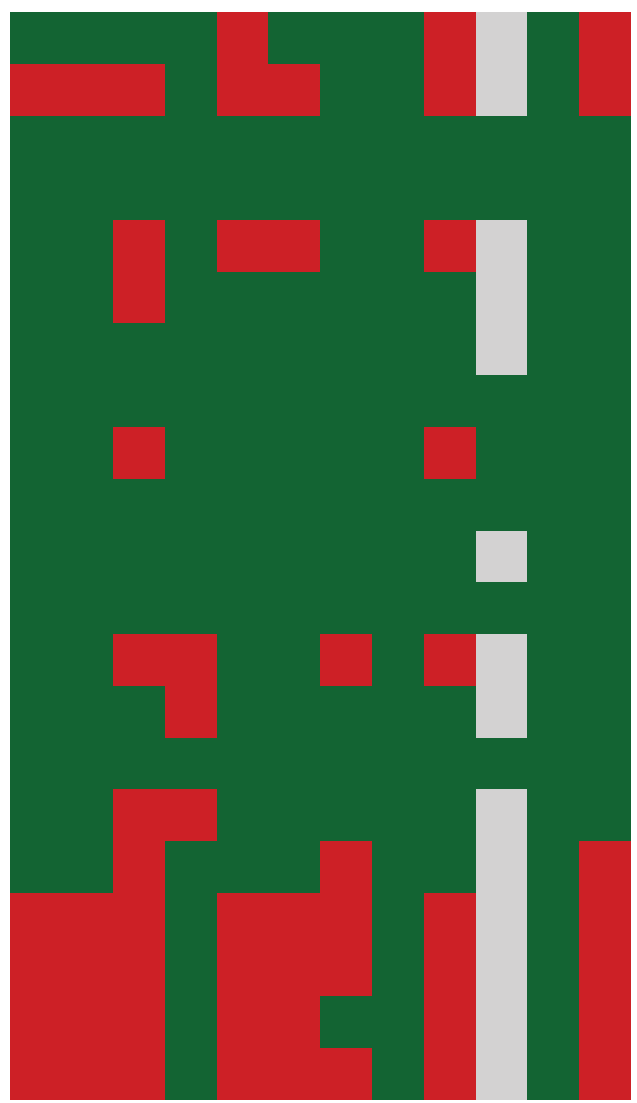

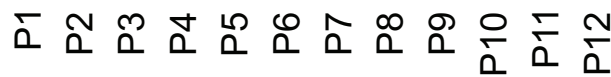

detected

not detected

nothing reported
O. mykiss

A. simplex

L. acidophilus

L. delbrueckii

A. flavus

AfdsV

E. coli

Norwalk virus

M. colombiense

S. enterica

L. monocytogenes

F. nucleatum

P. libanensis

B. pseudomallei

B. fragilis

ASFV

phiX174

B. malayi

C. remanei

D. rerio

S. japonicus

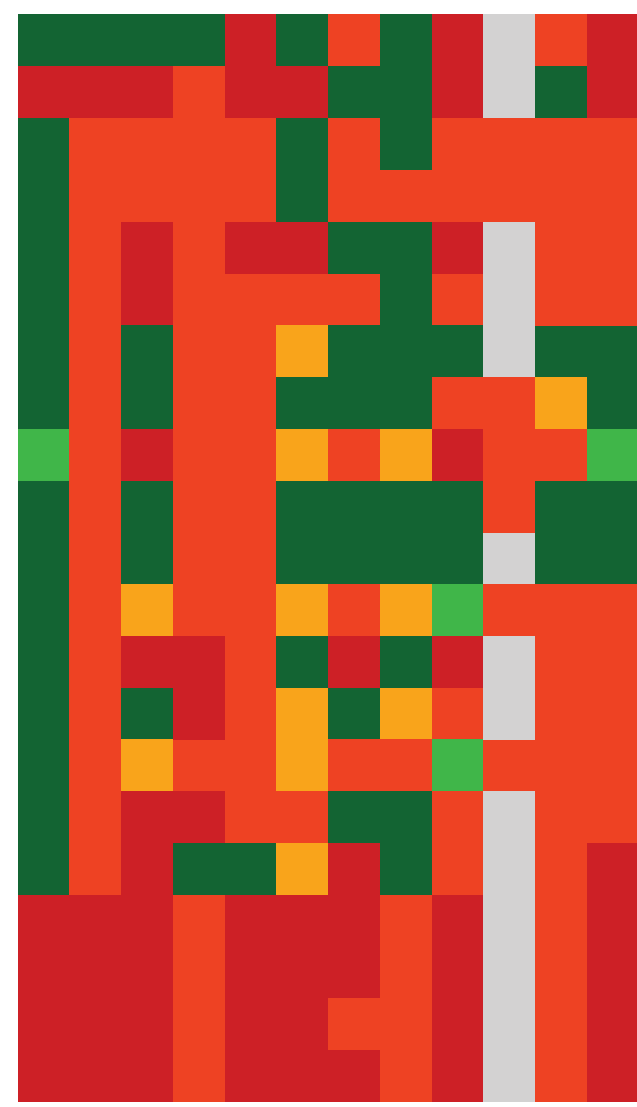

\section{๘ேヘ}

\section{rated as expected rated more serious rated less serious}

detected, not assessed

not detected

nothing reported

FIGURE 4 | Summary of the read-assignment tables (A), and assessment and interpretation of the assignments (B). Heatmap showing the positive (comprised species detected; green) and negative (comprised species NOT detected; red) results of the software analyses. The results shown for participants are based on their uploaded read assignment tables, except for P10, for which the results are derived from their summary table and assessment (A). Heatmap summarizing assessments of the detected species by the participants (B). Sequences of the taxa labeled gray (Brugia malayi, Caenorhabditis remanei, Danio rerio, and Scomber japonicus) were downloaded unintentionally as part of the Anisakis sequence dataset.

assessment and the background of the participant (compare Table 2). Though most species were detected (Figure 4A), the overall result was suboptimal (see summary in Figure 4B). For this assessment, the participants' assessments were compared with the expected categorization of the respective species (compare Table 1). The reasons for the observed deviations may be diverse, located both at the technical level and at the individual experiences of the personnel.

At the technical level, unsuitable parametrization of the analysis may be a possible cause for missing important species from the result the diagnostician gets for the assessment, i.e., arbitrary thresholds for taking detected species into consideration. For instance, thresholds set for read numbers assigned to a single species can prevent detection, as was the case with P5 (reporting a minimum read number of 500 for each individual species) failing to recognize the African swine fever virus (15 reads) and the Aspergillus foetidus dsRNA mycovirus (107 reads). Moreover, minimum genome coverage of detected species, or too stringent cut-offs for the identity of reads with reference sequences may prevent species from appearing in the table of the detected species. Another technical issue was the use of incomplete databases for taxonomic binning (see above).

With regard to the individual experience of the personnel, a number of different reasons could be considered. In two cases (P3 and P11), the assessment of Norovirus was based on the detected viral strain, which was closest to murine strains and therefore assessed as "no human pathogen". However, indications of potential pathogens should always be followed 
up, because of the possibility of detecting a modified or novel pathogen with only weak relationship with the closest known relative in the database. In other cases, assessment of the software output by personnel not trained for this task (e.g., assessment by bioinformaticians instead of microbiologists, physicians, or veterinarians), insufficient awareness of the impact of certain species due to insufficient training, or maybe unsatisfactory consideration due to time constraints may have caused the result.

\section{CONCLUSION}

The dry-lab part of this ring-trial showed that despite the abovementioned shortcomings in some analyses (namely usage of incomplete databases or unsuitable data pre-processing), overall the used software appears to have matured over the last years to allow for the correct identification of the majority of organisms represented in a metagenomics dataset. However, for a truly beneficial effect of diagnostic metagenomics for the detection of potentially present pathogens, it is especially necessary to put more effort into the training for the assessment and interpretation of the results delivered by the different software pipelines for the analysis of metagenomics data.

Two additional points should be stressed. First, in this proficiency test dataset, we included African swine fever virus and Escherichia virus phiX174 sequences as within run contaminants, which only three and four participants, respectively, correctly assessed. Noteworthy, the same effect frequently occurs in real sequencing runs [Illumina, 2013 (between runs), Sinha et al., 2017; Illumina, 2017 (within run)]. Therefore, knowledge of the content of samples from the same and previous runs might be necessary to take into account, information that was not available for this proficiency test. Most importantly, however, awareness to the problem needs to be raised. Second, the interaction between the different sectors (human, veterinary, and food) and the disciplines within these, e.g., virology, bacteriology, parasitology, needs to be strengthened. This must necessarily include enhanced awareness of the pathogens of importance for other sectors and disciplines, including reporting to the respective colleagues in case relevant pathogens are detected.

\section{DATA AVAILABILITY STATEMENT}

The datasets presented in this study can be found in online repositories. The names of the repository/repositories and accession number(s) can be found below: data are available from https://www.ebi.ac.uk/ebisearch/overview.ebi/about under accession PRJEB37463.

\section{REFERENCES}

Bolger, A. M., Lohse, M., and Usadel, B. (2014). Trimmomatic: a flexible trimmer for Illumina sequence data. Bioinformatics 30, 2114-2120. doi: 10 . 1093/bioinformatics/btu170

Boratyn, G. M., Schäffer, A. A., Agarwala, R., Altschul, S. F., Lipman, D. J., and Madden, T. L. (2012). Domain enhanced lookup time accelerated BLAST. Biol. Direct. 7:12. doi: 10.1186/1745-6150-7-12

\section{AUTHOR CONTRIBUTIONS}

$\mathrm{DH}$ and $\mathrm{CW}$ conceived the study, analyzed data, and wrote the manuscript. DH created the artificial dataset. JG, $A B$, JM, SMa, RE, CD, ST, IC, SMo, MJ, TP, RH, SP, ML, MH, $\mathrm{AW}, \mathrm{PC}, \mathrm{LC}, \mathrm{MT}, \mathrm{JS}, \mathrm{CS}, \mathrm{AD}, \mathrm{AN}$, and $\mathrm{MB}$ analyzed the artificial dataset and assessed the results. All authors revised and approved the manuscript.

\section{FUNDING}

This research was supported by the EU funded project COMPARE (Grant Agreement No. 643476). JG was supported by a grant of the German Federal Ministry of Education within the framework of the project Ess-B.A.R. (FKZ 13N13982).

\section{ACKNOWLEDGMENTS}

We thank Simone M. Cacciò for helpful discussions regarding eukaryotic microorganisms.

\section{SUPPLEMENTARY MATERIAL}

The Supplementary Material for this article can be found online at: https://www.frontiersin.org/articles/10.3389/fmicb. 2020.575377/full\#supplementary-material

Supplementary Figure 1 | Specificities of read assignments calculated from the complete read set based on the species assignments. Sequences of the taxa labeled gray (Brugia malayi, Caenorhabditis remanei, Danio rerio, and Scomber japonicus) were downloaded unintentionally as part of the Anisakis sequence dataset.

Supplementary Figure 2 | Correct classification rates of read assignments calculated from the complete read set based on the species assignments. Sequences of the taxa labeled gray (Caenorhabditis remanei, Brugia malayi, Danio rerio, and Scomber japonicus) were downloaded unintentionally as part of the Anisakis sequence dataset.

Supplementary Figure 3 | Negative predictive values of read assignments calculated from the complete read set based on the species assignments. Sequences of the taxa labeled gray (Caenorhabditis remanei, Brugia malayi, Danio rerio, and Scomber japonicus) were downloaded unintentionally as part of the Anisakis sequence dataset.

Supplementary Materials and Methods | Emails with requirements to participants and details on the calculation of sensitivity, specificity, correct classification rate, positive predictive value, and negative predictive value are presented.

Summary and Assessment of the Participants | All summary and assessment files sent in by the participants are included.

Brinkmann, A., Andrusch, A., Belka, A., Wylezich, C., Höper, D., Pohlmann, A., et al. (2019). Proficiency testing of virus diagnostics based on bioinformatics analysis of simulated in silico high-throughput sequencing datasets. J. Clin. Microbiol. 57:e00466-19. doi: 10.1128/JCM.00466-19

Buchfink, B., Xie, C., and Huson, D. H. (2015). Fast and sensitive protein alignment using DIAMOND. Nat. Methods 12, 59-60. doi: 10.1038/nmeth.3176

Chen, S., Zhou, Y., Chen, Y., and Gu, J. (2018). fastp: an ultra-fast all-in-one FASTQ preprocessor. Bioinformatics 34, i884-i890. doi: 10.1093/bioinformatics/bty560 
Cottier, F., Srinivasan, K. G., Yurieva, M., Liao, W., Poidinger, M., Zolezzi, F., et al. (2018). Advantages of meta-total RNA sequencing (MeTRS) over shotgun metagenomics and amplicon-based sequencing in the profiling of complex microbial communities. NPJ Biofilms Microbiomes. 4:2. doi: 10.1038/s41522017-0046-x

Herbig, A., Maixner, F., Bos, K. I., Zink, A., Krause, J., and Huson, D. H. (2016). MALT: fast alignment and analysis of metagenomic DNA sequence data applied to the Tyrolean Iceman. bioRxiv [Preprint]. doi: 10.1101/050559

Höper, D., Mettenleiter, T. C., and Beer, M. (2016). Metagenomic approaches to identifying infectious agents. Rev. Sci. Tech. 35, 83-93. doi: 10.20506/rst.35.1. 2419

Huang, W., Li, L., Myers, J. R., and Marth, G. T. (2012). ART: a nextgeneration sequencing read simulator. Bioinformatics 28, 593-594. doi: 10 . 1093/bioinformatics/btr708

Huson, D. H., Beier, S., Flade, I., Górska, A., El-Hadidi, M., Mitra, S., et al. (2016). MEGAN community edition - Interactive exploration and analysis of large-scale microbiome sequencing data. PLoS Comput. Biol. 12:e1004957. doi: 10.1371/journal.pcbi.1004957

Illumina (2013). Reducing Run-To-Run Carryover on the MiSeq Using Dilute Sodium Hypochlorite Solution. Illumina: San Diego, CA.

Illumina (2017). NextSeq ${ }^{T M} 550 D x$ Instrument. Document \# 1000000043133 v00. Illumina: San Diego, CA.

Junier, T., Huber, M., Schmutz, S., Kufner, V., Zagordi, O., Neuenschwander, S., et al. (2019). Viral metagenomics in the clinical realm: lessons learned from a Swiss-wide ring trial. Genes 655:10. doi: 10.3390/genes1009 0655

Kirstahler, P., Bjerrum, S. S., Friis-Møller, A., la Cour, M., Aarestrup, F. M., Westh, H., et al. (2018). Genomics-based identification of microorganisms in human ocular body fluid. Sci. Rep. 8:4126. doi: 10.1038/s41598-018-22416-4

Koutsoumanis, K., Allende, A., Alvarez-Ordoñez, A., Bolton, D., Bover-Cid, S., Chemaly, M., et al. (2019). Scientific Opinion on the whole genome sequencing and metagenomics for outbreak investigation, source attribution and risk assessment of food-borne microorganisms. EFSA J. 17:5898. doi: 10.2903/j.efsa. 2019.5898

Langmead, B., and Salzberg, S. L. (2012). Fast gapped-read alignment with Bowtie 2. Nat. Methods 9, 357-359. doi: 10.1038/nmeth.1923

$\mathrm{Li}, \mathrm{H}$. (2013). Aligning sequence reads, clone sequences and assembly contigs with BWA-MEM. arXiv [Preprint]. Available online at: https://arxiv.org/abs/1303. 3997 (accessed April 25, 2018).

Martin, M. (2011). Cutadapt removes adapter sequences from high-throughput sequencing reads. EMBnet J. 17, 10-12. doi: 10.14806/ej.17.1.200

Menzel, P., Ng, K. L., and Krogh, A. (2016). Fast and sensitive taxonomic classification for metagenomics with Kaiju. Nat. Commun. 7:11257. doi: 10 . 1038/ncomms11257

Petersen, T. N., Lukjancenko, O., Thomsen, M., Sperotto, M. M., Lund, O., Aarestrup, F. M., et al. (2017). MGmapper: reference based mapping and taxonomy annotation of metagenomics sequence reads. PLoS One 12:e176469. doi: 10.1371/journal.pone.0176469

R Core Team (2019). R: A Language and Environment for Statistical Computing. Vienna: R Foundation for Statistical Computing.
Salter, S. J., Cox, M. J., Turek, E. M., Calus, S. T., Cookson, W. O., Moffatt, M. F., et al. (2014). Reagent and laboratory contamination can critically impact sequence-based microbiome analyses. BMC Biol. 12:87. doi: 10.1186/s12915014-0087-z

Scheuch, M., Höper, D., and Beer, M. (2015). RIEMS: a software pipeline for sensitive and comprehensive taxonomic classification of reads from metagenomics datasets. BMC Bioinf. 16:69. doi: 10.1186/s12859-015-0503-6

Scholz, M., Ward, D., Pasolli, E., Tolio, T., Zolfo, M., Asnicar, F., et al. (2016). Strain-level microbial epidemiology and population genomics from shotgun metagenomics. Nat. Methods 13, 435-438. doi: 10.1038/nmeth.3802

Schulz, M. H., Zerbino, D. R., Vingron, M., and Birney, E. (2012). Oases: robust de novo RNA-seq assembly across the dynamic range of expression levels. Bioinformatics 28, 1086-1092. doi: 10.1093/bioinformatics/bts094

Sinha, R., Stanley, G., Gulati, G. S., Ezran, C., Travaglini, K. J., Wie, E., et al. (2017). Index switching causes "spreading-of-signal" among multiplexed samples in Illumina HiSeq 4000 DNA Sequencing. BioRxiv [Preprint]. Available online at: https://www.biorxiv.org/content/early/2017/04/09/125724 (accessed June 23, 2020).

Tausch, S. H., Loka, T. P., Schulze, J. M., Andrusch, A., Klenner, J., Dabrowski, P. W., et al. (2018). PathoLive - Real time pathogen identification from metagenomic Illumina datasets. BioRxiv [Preprint]. doi: 10.1101/402370

Wingett, S. W., and Andrews, S. (2018). FastQ screen: a tool for multigenome mapping and quality control. F1000Research 7:1338. doi: 10.12688/ f1000research.15931.2

Wood, D. E., and Salzberg, S. L. (2014). Kraken: ultrafast metagenomic sequence classification using exact alignments. Genome Biol. 15:R46. doi: 10.1186/gb2014-15-3-r46

Wylezich, C., Papa, A., Beer, M., and Höper, D. (2018). A versatile sample processing workflow for diagnostic metagenomics. Sci. Rep. 8:13108. doi: 10. 1038/s41598-018-31496-1

Zamperin, G., Lucas, P., Cano, I., Ryder, D., Abbadi, M., Stone, D., et al. (2019). Sequencing of animal viruses: quality data assurance for NGS bioinformatics. Virol. J. 16:140. doi: 10.1186/s12985-019-1223-8

Zolfo, M., Tett, A., Jousson, O., Donati, C., and Segata, N. (2017). MetaMLST: multi-locus strain-level bacterial typing from metagenomic samples. Nucleic Acids Res. 45:e7. doi: 10.1093/nar/gkw837

Conflict of Interest: The authors declare that the research was conducted in the absence of any commercial or financial relationships that could be construed as a potential conflict of interest.

Copyright (c) 2020 Höper, Grützke, Brinkmann, Mossong, Matamoros, Ellis, Deneke, Tausch, Cuesta, Monzón, Juliá, Petersen, Hendriksen, Pamp, Leijon, Hakhverdyan, Walsh, Cotter, Chandrasekaran, Tay, Schlundt, Sala, De Cesare, Nitsche, Beer and Wylezich. This is an open-access article distributed under the terms of the Creative Commons Attribution License (CC BY). The use, distribution or reproduction in other forums is permitted, provided the original author(s) and the copyright owner(s) are credited and that the original publication in this journal is cited, in accordance with accepted academic practice. No use, distribution or reproduction is permitted which does not comply with these terms. 